\title{
Adaptive dynamic programming algorithm for uncertain nonlinear switched systems
}

\author{
Dao Phuong Nam ${ }^{1}$, Nguyen Hong Quang ${ }^{2}$, Nguyen Nhat Tung ${ }^{3}$, Tran Thi Hai Yen ${ }^{4}$ \\ ${ }^{1}$ School of Electrical Engineering, Hanoi University of Science and Technology, \\ Bách Khoa, Hai Bà Trung, Hà Noi, Vietnam \\ ${ }^{2,4}$ Thai Nguyen University of Technology, So 666 D. 3/2, P, Thành pho Thái Nguyên, Thái Nguyên, Vietnam \\ ${ }^{3}$ Electric Power University, 235 Hoàng Quoc Viet, Co Nhue, Tu Liêm, Hà Noi 129823, Vietnam
}

\begin{abstract}
Article Info
Article history:

Received Feb 2, 2020

Revised Dec 15, 2020

Accepted Jan 10, 2021

Keywords:

Adaptive dynamic

ABSTRACT

This paper studies an approximate dynamic programming (ADP) strategy of a group of nonlinear switched systems, where the external disturbances are considered. The neural network $(\mathrm{NN})$ technique is regarded to estimate the unknown part of actor as well as critic to deal with the corresponding nominal system. The training technique is simultaneously carried out based on the solution of minimizing the square error Hamilton function. The closed system's tracking error is analyzed to converge to an attraction region of origin point with the uniformly ultimately bounded (UUB) description. The simulation results are implemented to determine the effectiveness of the ADP based controller.
\end{abstract}

programming

HJB equation

Lyapunov

Neural networksstability

Nonlinear switched systems

Corresponding Author:
This is an open access article under the CC BY-SA license.

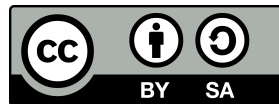

Nguyen Hong Quang

Thai Nguyen University of Technology,

So 666 D. 3/2, P, Thành pho Thái Nguyên, Thái Nguyên, Vietnam

Email: quang.nguyenhong@ tnut.edu.vn

\section{INTRODUCTION}

It is worth noting that many systems in industry can be described by switched system such as DCDC converter [1]-[3], H-bridge inverter [4], multilevel inverter [5], photovoltaic inverter [6]. Although many different approaches for switched systems have been proposed, e.g., switching-delay tolerant control [7], classical nonlinear control [8]-[12], the optimization approaches with the advantage of mentioning the input/state constraint has not been mentioned much. The approaches of fuzzy and neural network as well as ANN, particle swarm optimization (PSO) technique were investigated in several different systems such as photovoltaic inverter, transmission line. [13]-[17].

Adaptive dynamic programming has been considered in many situations, such as nonlinear continuous time systems [18], actuator saturation [19], linear systems [20]-[22], output constraint [23]. In the case of nonlinear systems, the algorithm should be implemented based on Neural Networks (NNs). However, Kronecker product was employed in linear systems. Furthermore, the data driven technique should to be mentioned to compute the actor/critic precisely. It should be noted that the robotic systems has been controlled by ADP algorithm [24]-[25].

Our work proposed the solution of adaptive dynamic programming in nonlinear perturbed switching systems based on the neural networks. The consideration of the Halminton function enables us obtaining the learning technique of these neural networks. The UUB stability of closed system is analyzed and simulation results illustrate the high effectiveness of given controller. 


\section{PROBLEM STATEMENTS}

Consider the following uncertain nonlinear continuous time switched systems of the form:

$$
\frac{d}{d t} \xi(t)=f_{i}(\xi(t))+g_{i}(\xi(t))(u+\Delta(\xi, t))
$$

where $\xi(t) \in \Omega_{x} \in \mathbb{R}^{n}$ denotes the state variables and $u(t) \in \Omega_{u} \in \mathbb{R}^{m}$ describes the control variables. The function $\beta:[0,+\infty) \mapsto \Omega=\{1,2, \ldots, l\}$ is a information of switching processing, which is known as a function with many continuous piecewise depending on time, and $l$ is the subsystems number. $f_{i}(\xi)$ are uncertain smooth vector functions with $f_{i}(0)=0 . g_{i}(\xi)$ are mentioned as smooth vector functions with the property $G_{\min } \leqslant\left\|g_{i}(\xi)\right\| \leqslant G_{\max }$. The switching index $\beta(t)$ is unknown.

Assumption 1: $\Delta(\xi, t)$ is bounded by a certain function $\varrho(\xi)$ as $\|\Delta(\xi, t)\| \leqslant \varrho(\xi)$

Consider the cost function connected with the uncertain switched system (1):

$$
J(\xi, u)=\int_{t}^{\infty} r(\xi(\tau), u(\tau)) d \tau
$$

where $r(\xi, u)=\xi^{T} Q \xi+u^{T} R u$ and $Q=Q^{T}>0 ; R=R^{T}>0$.

The main purpose is to achieve the state feedback control design and give the upper bound term to guarantee the closed systems under this controller is robustly stable. Additionally, the performance index (2) is bounded as $J \leq K(\xi, u) \leq M$.

Definition: The term $K(u)$ is given by the appropriate performance index. As a result, the control input $u^{*}=\arg \min _{u \in \Omega_{u}} K(\xi, u)$ is mentioned as the optimal appropriate performance index method.

\section{CONTROL DESIGN}

The obtained nominal system after eliminating the disturbance in switched system (3) is described by:

$$
\frac{d}{d t} \xi=f_{i}(\xi)+g_{i}(\xi) u
$$

The performance index of system (3) is modified as (4)

$$
Q_{1}(\xi, u)=\int_{t}^{\infty}\left[r(\xi, u)+\gamma(\rho(\xi))^{2}\right] d \tau
$$

We prove that $Q_{1}(\xi, u)$ with $\gamma \geqslant\|R\|$ is the one of appropriate performance indexes of dynamical system (1). Define: $V^{*}(t)=\min _{u \in \Omega_{u}} Q_{1}(\xi, u)$, we have (5)

$$
V^{*}(t)=\min _{u \in \Omega_{u}} \int_{t}^{\infty}\left\{r(\xi, u)+\gamma \rho^{2}(\xi)\right\} d \lambda
$$

based on nominal system and cost function (4), it leads to Halminton function as (6)

$$
H\left(\xi, u, V^{*}\right)=r(\xi, u)+\gamma \rho^{2}(\xi)+\left(\frac{\partial V^{*}}{\partial \xi}\right)^{T}\left(f_{i}(\xi)+g_{i}(\xi) u\right)
$$

by using optimality principle, the optimal control input can be obtained as (7).

$$
u^{*}(\xi)=-\frac{1}{2} R^{-1}\left(g_{i}(\xi)\right)^{T} \frac{\partial V^{*}}{\partial \xi}
$$

We continue to utilize this control law (7) for nonlinear continuous SW system (1) and obtain that:

Theorem 1: The system (1) under the controller $u^{*}(\xi)=-\frac{1}{2} R^{-1}\left(g_{i}(\xi)\right)^{T} \frac{\partial V^{*}}{\partial \xi}$ is stable with the associated Lyapunov function candidate: 


$$
V(t)=\int_{t}^{\infty}\left\{r(\xi, u)+\gamma \varrho^{2}(\xi)\right\} d \lambda
$$

where $\gamma \geqslant\|R\|$. that (9):

Proof: Taking the derivative of $V$ under the control input $u(\xi)=-\frac{1}{2} R^{-1}\left(g_{i}(\xi)\right)^{T} \nabla V^{*}$, we imply

$$
\frac{d}{d t} V=-\xi^{T} Q \xi-\left(\gamma \varrho^{2}(\xi)-\Delta(\xi, t)^{T} R \Delta(\xi, t)\right)-(u+\Delta(\xi, t))^{T} R(u+\Delta(\xi, t))
$$

It is able to conclude that (10):

$$
\dot{V}(t) \leqslant-\xi^{T} Q \xi
$$

Therefore, the system (1) is robustly stable. However, it is impossible to solve directly HJB equation. Hence, the optimal performance index $V^{*}$ for system (3) can be described based on a NN as (11)

$$
V^{*}=w^{T} \sigma(\xi)+\varepsilon(\xi)
$$

where $\sigma(x): R^{n} \rightarrow R^{N} ; \sigma(0)=0, w \in R^{N}$ is the NN constant weight vector. $\sigma(x)$ can be found to guarantee that when $N \rightarrow \infty$, we have: $\varepsilon(\xi) \rightarrow 0$ and $\nabla \varepsilon(\xi) \rightarrow 0$, so for fixed $N$, we can assume that:

Assumption 2: $\|\varepsilon(\xi)\| \leqslant \varepsilon_{\max } ;\|\nabla \varepsilon(\xi)\| \leqslant \nabla \varepsilon_{\max } ; \nabla \sigma_{\min } \leqslant\|\nabla \sigma(\xi)\| \leqslant \nabla \sigma_{\max } ;\|w\| \leqslant w_{\max }$.

Combining two formulas (10) and (11) we imply (12)

$$
H\left(\xi, u^{*}, V^{*}\right)=\xi^{T} Q \xi+\lambda \varrho^{2}(\xi)+\left(\nabla V^{*}\right)^{T} f_{i}(\xi)-\frac{1}{4}\left(\nabla V^{*}\right)^{T} g_{i}(\xi) R^{-1} g_{i}(\xi)^{T}\left(\nabla V^{*}\right)=0
$$

Formula (19) leads to (13).

$$
\nabla V^{*}=(\nabla \sigma(\xi))^{T} w+\nabla \varepsilon(\xi)
$$

Obtain the description as (14).

$$
e_{N N}=-\nabla \varepsilon(\xi)^{T}\left(f_{i}(\xi)+g_{i}(\xi) u^{*}\right)+\frac{1}{4} \nabla \varepsilon(\xi)^{T} g_{i}(\xi) R^{-1} g_{i}(\xi)^{T} \nabla \varepsilon(\xi)
$$

It follows that $e_{N N}$ converges uniformly to zero as $N \rightarrow \infty$. For each number $N, e_{N N}$ is bounded on a region as $e_{N N} \leqslant e_{\max }$. Under the structure of ADP-based controller, a critic NN is computed as (15).

$$
\hat{V}=\hat{w}^{T} \sigma(\xi)=\sigma(\xi)^{T} \hat{w} ; \hat{u}=-\frac{1}{2} R^{-1}\left(g_{i}(\xi)\right)^{T} \nabla \hat{V}
$$

It is able to achieve that:

$$
e_{H J B}=\xi^{T} Q \xi+\lambda \varrho^{2}(\xi)+\hat{w}^{T} \nabla \sigma(\xi) f_{i}(\xi)-\frac{1}{4} \hat{w}^{T} \nabla \sigma(\xi) g_{i}(\xi) R^{-1} g_{i}(\xi)^{T} \nabla \sigma(\xi)^{T} \hat{w}
$$

The training law is handled based on a steepest descent method:

$$
\frac{d}{d t} \widehat{w}=-\alpha \frac{\partial E}{\partial \widehat{w}}
$$

with $E=\frac{1}{2} e_{H J B}^{T} e_{H J B}$.

Remark 1: The weight $\widehat{w}$ is trained to minimize the network error part $G=\frac{1}{2} e_{H J B}^{T} e_{H J B}$. This result is obtained from (18).

$$
\frac{\partial G}{\partial t}=-\alpha\left(\frac{\partial G}{\partial \widehat{w}}\right)^{2}
$$


Theorem 2: Consider the feedback controller in (15) and the critic weight is updated by (18), the weight estimate error $\tilde{w}=w-\hat{w}$ and the closed system's state vector $x(t)$ are uniformly ultimately bounded (UUB).

Proof: Let's choose the Lyapunov function:

$$
V(t)=V_{1}(t)+V_{2}(t), \text { where: } V_{1}(t)=\frac{1}{2 \alpha} \tilde{w}(t)^{T} \tilde{w}(t), V_{2}(t)=V^{*}
$$

Using the Assumption 3: $\left\|f_{i}(\xi)+g_{i}(\xi) u^{*}\right\| \leqslant \rho_{\max }$ and the definition: $\rho_{i}=f_{i}(\xi)+g_{i}(\xi) u^{*} ; G_{i}=g_{i}(\xi) R^{-1} g_{i}(\xi)^{T} ; \nabla \sigma=\nabla \sigma(\xi) ; \nabla \varepsilon=\nabla \varepsilon(\xi)$. Taking the derivative of $V_{1}(t)$, we imply that:

$$
\begin{gathered}
\dot{V}_{1}(t)=-\tilde{w}^{T}\left(-e_{N N}+\tilde{w}^{T} \nabla \sigma \mu_{i}+\frac{1}{2} \tilde{w}^{T} \nabla \sigma G_{i} \nabla \varepsilon+\frac{1}{4} \tilde{w}^{T} \nabla \sigma G_{i} \nabla \sigma^{T} \tilde{w}\right) \\
\nabla \sigma(x)\left(\mu_{i}+\frac{1}{2} G_{i}\left(\nabla \sigma^{T} \tilde{w}+\nabla \varepsilon\right)\right)
\end{gathered}
$$

It leads to the estimation: $\dot{V}_{1}(t) \leqslant-\pi_{1}$. For the term $V_{2}(t)$, from (20) we have (21).

$$
\begin{gathered}
\dot{V}_{2}=\left(\nabla V^{*}\right)^{T}\left(f_{i}+g_{i}(\hat{u}+\Delta)\right)=-\left(\xi^{T} Q \xi+\lambda \rho^{2}(\xi)\right)-\frac{1}{4}\left(\nabla V^{*}\right)^{T} \\
g_{i} R^{-1} g_{i}^{T}\left(\nabla V^{*}\right)+\frac{1}{2}\left(\nabla V^{*}\right)^{T} g_{i} R^{-1} g_{i}^{T}\left(\nabla \sigma(\xi)^{T} \tilde{w}+\nabla \varepsilon(\xi)\right)+\left(\nabla V^{*}\right)^{T} g_{i} \Delta
\end{gathered}
$$

Assume that $\rho(\xi)=\varpi\|\xi\|$. From (40) we have (22).

$$
\dot{V}_{2} \leqslant-\left(\lambda_{\min }(Q)+\lambda \varpi\right)\|\xi\|^{2}+\theta^{2}
$$

with $\theta^{2}=-\frac{1}{4}\left(\nabla V^{*}\right)^{T} g_{i} R^{-1} g_{i}^{T}\left(\nabla V^{*}\right)+\frac{1}{2}\left(\nabla V^{*}\right)^{T} g_{i} R^{-1} g_{i}^{T}\left(\nabla \sigma(x)^{T} \tilde{w}+\nabla \varepsilon(x)\right)+\left(\nabla V^{*}\right)^{T} g_{i} \Delta$.

Based on the two above assumptions, we have (23).

$$
\begin{gathered}
\theta^{2} \leqslant \frac{1}{4}\left(w_{\max } \nabla \sigma_{\max }+\nabla \varepsilon_{\max }\right)^{2} g_{\max }^{2} \lambda_{\max }\left(R^{-1}\right)+\frac{1}{2}\left(\vartheta \nabla \sigma_{\max }+\nabla \varepsilon_{\max }\right)^{2} g_{\max }^{2} \lambda_{\max }\left(R^{-1}\right) \\
+\left(w_{\max } \nabla \sigma_{\max }+\nabla \varepsilon_{\max }\right) g_{\max } \varpi\|x\|
\end{gathered}
$$

It is obvious that $\left(\lambda_{\min }(Q)+\lambda \varpi\right)\|x\|^{2}-\theta^{2} \geqslant \pi_{2}$ with $\pi_{2}>0$ and we obtain (24).

$$
\dot{V}_{2}(t) \leqslant-\pi_{2}
$$

Remark 2: The coefficients $\vartheta_{1} ; \vartheta_{2}$ can be chosen by renovating the $\mathrm{NN}$ of the optimal performance index. Moreover, for arbitrary switching index, after $\frac{V(0)}{\min \left(\pi_{1} ; \pi_{2}\right)}$ the variable $\|\xi\|$ and $\|\tilde{w}\|$ tend to the accurate domains. The ADP controller $\hat{u}$ is proposed in (15), which tends to the neighborhood of $u^{*}$.

Proof: The deviation of control input is estimated as (25).

$$
\begin{gathered}
\left\|\hat{u}-u^{*}\right\|=\frac{1}{2}\left\|R^{-1}\left(g_{i}(\xi)\right)^{T}\left((\nabla \sigma(\xi))^{T} \tilde{w}+\nabla \varepsilon(\xi)\right)\right\| \\
\leqslant \frac{1}{2} \lambda_{\max }\left(R^{-1}\right) \cdot G_{\max } \cdot\left(\nabla \sigma_{\max } \cdot v_{1}+\nabla \varepsilon_{\max }\right)=\vartheta_{3}
\end{gathered}
$$

Thus the proof is completed. 


\section{SIMULATION RESULTS}

In this section, we consider the simulations to validate the performance of the established control scheme: Let $N=2$ and the subsystems of the switched system are (26) and (27).

$$
\begin{aligned}
& \left\{\begin{array}{l}
\dot{x}_{1}=-x_{1}^{3}-2 x_{2}+\left(u+\Delta_{1}(x, t)\right) \\
\dot{x}_{2}=x_{1}+0.5 \cos \left(x_{1}^{2}\right) \sin \left(x_{2}^{3}\right)-\left(u+\Delta_{1}(x, t)\right)
\end{array}\right. \\
& \left\{\begin{array}{l}
\dot{x}_{1}=-x_{1}^{5} \sin \left(x_{2}\right)+\left(u+\Delta_{2}(x, t)\right) \\
\dot{x}_{2}=\frac{1}{2} x_{1}-\cos \left(x_{1}\right) \cos \left(x_{2}^{3}\right)-\left(u+\Delta_{2}(x, t)\right)
\end{array}\right.
\end{aligned}
$$

The initial state vectors can be chosen as (28).

$$
x(0)=\left[\begin{array}{ll}
5 & -5
\end{array}\right]^{T}
$$

Choosing that the parameter matrices: $R=\left[\begin{array}{cc}2 & 0 \\ 0 & 2\end{array}\right] ; Q=\left[\begin{array}{cc}1 & 0 \\ 0 & 3\end{array}\right] ; \alpha=0.1 ; \lambda=5$.

The simulation results shown in Figure 1 and Figure 2 validate the effectiveness of proposed algorithm.

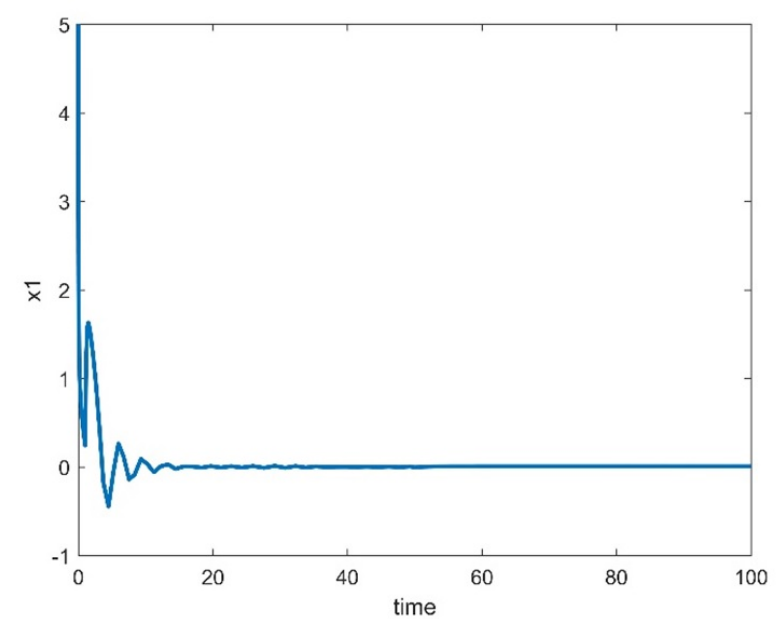

Figure 1. The response of $\mathrm{x}_{2}$

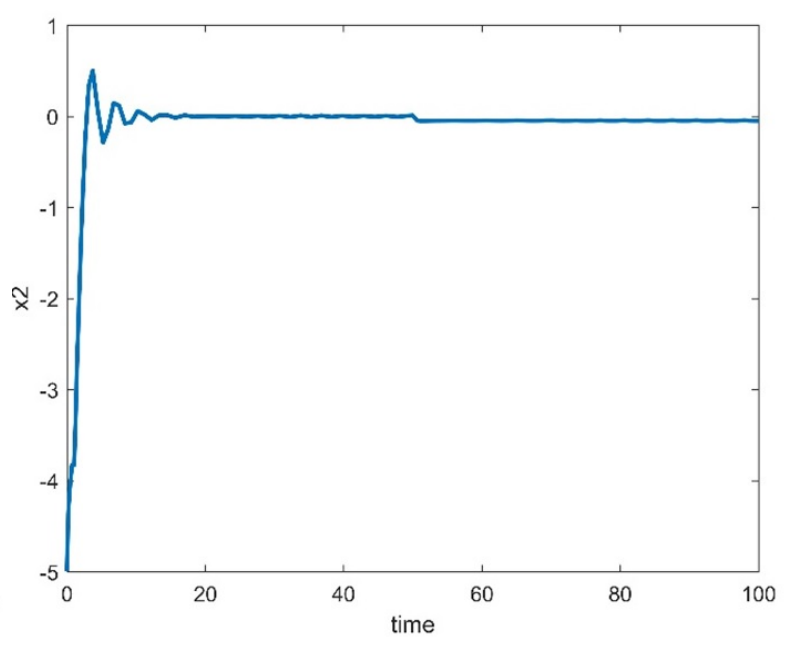

Figure 2. The response of $x_{2}$

\section{CONCLUSION}

This paper has investigated the ADP problem of switched nonlinear systems under the external disturbance. We consider previously for nominal system by eliminating the disturbance, then using classical nonlinear control technique. The neural networks have been designed to estimate the actor and critic NN of iteration. It is possible to develop the learning algorithm with simultaneous tuning. Finally, UUB description of the closed system is guaranteed under this work.

\section{ACKNOWLEDGEMENT}

This research was supported by Research Foundation funded by Thai Nguyen University of Technology.

\section{REFERENCES}

[1] Vu, Tran Anh and Nam, Dao Phuong and Huong, Pham Thi Viet, "Analysis and control design of transformerless high gain, high efficient buck-boost DC-DC converters," in 2016 IEEE International Conference on Sustainable Energy Technologies (ICSET), Hanoi, 2016, pp. 72-77, doi: 10.1109/ICSET.2016.7811759. 
[2] Nam, Dao Phuong and Thang, Bui Minh and Thanh, Nguyen Truong, "Adaptive Tracking Control for a Boost DC-DC Converter: A Switched Systems Approach," in 2018 4th International Conference on Green Technology and Sustainable Development (GTSD), Ho Chi Minh City, 2018, pp. 702-705, doi: 10.1109/GTSD.2018.8595580.

[3] Thanh, Nguyen Truong and Sam, Pham Ngoc and Nam, Dao Phuong, "An Adaptive Backstepping Control for Switched Systems in presence of Control Input Constraint," in 2019 International Conference on System Science and Engineering (ICSSE), Dong Hoi, Vietnam, 2019, pp. 196-200, doi: 10.1109/ICSSE.2019.8823125.

[4] Panigrahi, Swetapadma and Thakur, Amarnath, "Modeling and simulation of three phases cascaded Hbridge grid-tied PV inverter," Bulletin of Electrical Engineering and Informatics (BEEI), vol. 8, no. 1, pp. 1-9, 2019, doi: 10.11591/eei.v8i1.1225.

[5] Devarajan, N and Reena, A, "Reduction of switches and DC sources in Cascaded Multilevel Inverter," Bulletin of Electrical Engineering and Informatics (BEEI), vol. 4, no. 3, pp. 186-195, 2015, doi: 10.11591/eei.v4i3.320.

[6] Venkatesan, $\mathrm{M}$ and Rajeshwari, $\mathrm{R}$ and Deverajan, $\mathrm{N}$ and Kaliyamoorthy, M, "Comparative study of three phase grid connected photovoltaic inverter using pi and fuzzy logic controller with switching losses calculation," International Journal of Power Electronics and Drive Systems (IJPEDS), vol. 7, no. 2, pp. 543-550, 2016.

[7] Zhang, Lixian and Xiang, Weiming, "Mode-identifying time estimation and switching-delay tolerant control for switched systems: An elementary time unit approach," Automatica, vol. 64, pp. 174-181, 2016, doi: 10.1016/j.automatica.2015.11.010.

[8] Yuan, Shuai and Zhang, Lixian and De Schutter, Bart and Baldi, Simone, "A novel Lyapunov function for a non-weighted L2 gain of asynchronously switched linear systems," Automatica, vol. 87, pp. 310-317, 2018, doi: 10.1016/j.automatica.2017.10.018.

[9] Xiang, Weiming and Lam, James and Li, Panshuo, "On stability and $\mathrm{H}$ control of switched systems with random switching signals," Automatica, vol. 95, pp. 419-425, 2018, doi: 10.1016/j.automatica.2018.06.001.

[10] Lin, Jinxing and Zhao, Xudong and Xiao, Min and Shen, Jingjin, "Stabilization of discrete-time switched singular systems with state, output and switching delays," Journal of the Franklin Institute, vol. 356, pp. 2060-2089, 2019, doi: 10.1016/j.jfranklin.2018.11.034.

[11] Briat, Corentin, "Convex conditions for robust stabilization of uncertain switched systems with guaranteed minimum and mode-dependent dwell-time," Systems \& Control Letters, vol. 78, pp. 63-72, 2015, doi: 10.1016/j.sysconle.2015.01.012.

[12] Lian, Jie and Li, Can, "Event-triggered control for a class of switched uncertain nonlinear systems," Systems \& Control Letters, vol. 135, pp. 1-5, 2020, doi: 10.1016/j.sysconle.2019.104592.

[13] Anyaka, Boniface O and Manirakiza, J Felix and Chike, Kenneth C and Okoro, Prince A, "Optimal unit commitment of a power plant using particle swarm optimization approach," International Journal of Electrical and Computer Engineering (IJECE), vol. 10, no.2, pp. 1135-1141, 2020, doi: 10.11591/ijece.v10i2.pp1135-1141.

[14] Devi, Palakaluri Srividya and Santhi, R Vijaya, "Introducing LQR-fuzzy for a dynamic multi area LFCDR model," International Journal of Electrical \& Computer Engineering, vol. 9, no. 2, pp. 861-874, 2019, doi: 10.11591/ijece.v9i2.pp861-874.

[15] Omar, Othman AM and Badra, Niveen M and Attia, Mahmoud A, "Enhancement of on-grid pv system under irradiance and temperature variations using new optimized adaptive controller," International Journal of Electrical and Computer Engineering (IJECE), vol. 8, no. 5, pp. 2650-2660, 2018, doi: 10.11591/ijece.v8i5.2650-2660.

[16] Sharma, Purva and Saini, Deepak and Saxena, Akash, "Fault detection and classification in transmission line using wavelet transform and ANN," Bulletin of Electrical Engineering and Informatics (BEEI), vol. 5, no. 3, pp. 284-295, 2016.

[17] Ilamathi, P and Selladurai, V and Balamurugan, K, "Predictive modelling and optimization of nitrogen oxides emission in coal power plant using Artificial Neural Network and Simulated Annealing," IAES International Journal of Artificial Intelligence (IJ-AI), vol. 1, no. 1, pp. 11-18, 2012.

[18] Vamvoudakis, Kyriakos G and Vrabie, Draguna and Lewis, Frank L, "Online adaptive algorithm for optimal control with integral reinforcement learning," International Journal of Robust and Nonlinear 
Control, vol. 24, no. 17, pp. 2686-2710, 2013, doi: 10.1002/rnc.3018.

[19] Bai, Weiwei and Zhou, Qi and Li, Tieshan and Li, Hongyi, "Adaptive reinforcement learning neural network control for uncertain nonlinear system with input saturation," IEEE transactions on cybernetics, vol. 50, no. 8, pp. 3433-3443, Aug. 2020, doi: 10.1109/TCYB.2019.2921057.

[20] Chen, Ci and Modares, Hamidreza and Xie, Kan and Lewis, Frank L and Wan, Yan and Xie, Shengli, "Reinforcement learning-based adaptive optimal exponential tracking control of linear systems with unknown dynamics," in IEEE Transactions on Automatic Control, vol. 64, no. 11, pp. 4423-4438, Nov. 2019, doi: 10.1109/TAC.2019.2905215.

[21] Vamvoudakis, Kyriakos G and Ferraz, Henrique, "Model-free event-triggered control algorithm for continuous-time linear systems with optimal performance," in Automatica, vol. 87, pp. 412-420, 2018, doi: 10.1016/j.automatica.2017.03.013.

[22] Gao, Weinan and Jiang, Yu and Jiang, Zhong-Ping and Chai, Tianyou, "Output-feedback adaptive optimal control of interconnected systems based on robust adaptive dynamic programming," Automatica, vol. 72, pp. 37-45, 2016, doi: 10.1016/j.automatica.2016.05.008.

[23] Zhang, Tianping and Xu, Haoxiang, "Adaptive optimal dynamic surface control of strict-feedback nonlinear systems with output constraints," International Journal of Robust and Nonlinear Control, vol. 30, no. 5, pp. 2059-2078, 2020, doi: 10.1002/rnc.4864.

[24] Wang, Ding and Mu, Chaoxu, "Adaptive-critic-based robust trajectory tracking of uncertain dynamics and its application to a spring-mass-damper system," IEEE Transactions on Industrial Electronics, vol. 65, no. 1, pp. 654-663, Jan. 2018, doi: 10.1109/TIE.2017.2722424.

[25] Wen, Guoxing and Ge, Shuzhi Sam and Chen, CL Philip and Tu, Fangwen and Wang, Shengnan, "Adaptive tracking control of surface vessel using optimized backstepping technique," IEEE transactions on cybernetics, vol. 49, no. 9, pp. 3420-3431, Sept. 2019, doi: 10.1109/TCYB.2018.2844177. 